Published in final edited form as:

Arch Womens Ment Health. 2017 October ; 20(5): 621-632. doi:10.1007/s00737-017-0726-9.

\title{
Pregnancy and postpartum antidepressant use moderates the effects of sleep on depression
}

\author{
Kristen C. Stone ${ }^{1,2}$, Amy L. Salisbury ${ }^{1,2}$, Cynthia L. Miller-Loncar ${ }^{1,2}$, Jennifer A. Mattera ${ }^{2}$, \\ Cynthia L. Battle ${ }^{1,2,3}$, Dawn M. Johnsen², Kevin E. O’Grady \\ ${ }^{1}$ Alpert Medical School of Brown University, Box G-A1, Providence, RI 02912, USA \\ ${ }^{2}$ Women \& Infants Hospital, 101 Dudley St., Providence, RI 02905, USA \\ ${ }^{3}$ Butler Hospital, 345 Blackstone Blvd., Providence, RI 02906, USA \\ ${ }^{4}$ Department of Psychology, University of Maryland, College Park, MD 20742, USA
}

\section{Abstract}

This study examined the course of antidepressant use, sleep quality, and depression severity from pregnancy through 6-month postpartum in women with and without a depressive disorder during pregnancy. Women $(N=215)$ were interviewed during pregnancy, 1- and 6-month postpartum. Mixed linear models were used to examine the longitudinal course and inter-relationships for the time-varying variables of antidepressant use, subjective sleep quality, and depression severity. Pregnant women with a depressive disorder who did not use antidepressants had more variable depression severity over time with improvements in depression severity by 6-month postpartum. In contrast, the depression severity of their medicated counterparts remained stable and high throughout. Pregnant women without a depressive disorder had worse sleep quality when using antidepressants compared with when they were not. Antidepressant use significantly strengthened the magnitude of the effect of sleep quality on depression severity in women with a depressive disorder during pregnancy. When prenatally depressed women use antidepressants, their sleep disturbance is more highly linked to depression severity than when they do not. Furthermore, antidepressants are not adequately treating the sleep disturbance of these women or their remitted counterparts, leaving both groups vulnerable to significant negative mental and physical health outcomes.

\section{Keywords}

Sleep; Depression; Postpartum; Pregnancy; Antidepressant medication

Depression in pregnancy is prevalent and associated with preeclampsia, preterm birth, abnormal bleeding, miscarriages, as well as other mental and physical health problems that extend into the postnatal period for mothers and their infants (Battle et al. 2006; Gavin et al. 2005; Grigoriadis et al. 2013b; Grote et al. 2010; Salisbury et al. 2016; Salisbury et al.

Kristen C. Stone, Kristen_Stone@brown.edu.

Conflict of interest The authors declare that they have no conflict of interest. 
2011). Sleep disturbance is not only a negative outcome of depression during pregnancy (Field et al. 2007; Okun et al. 2011) but also a risk factor for the development of depression during pregnancy (Okun et al. 2009a; Skouteris et al. 2008; Wu et al. 2014). Sleep disturbance and depressive symptoms may increase the risk for adverse pregnancy outcomes, particularly for women with existing depression (Field et al. 2006; Micheli et al. 2011). For example, in depressed samples, reduced sleep duration during pregnancy has been linked with lower birth weight, ostensibly dysregulating inflammatory processes (Okun et al. 2009b, 2013).

Although the first-line treatment for unipolar depression is antidepressant medication, (Cooper et al. 2007; Davidson 2010) evidence exists that, among women with a history of depression, being on an antidepressant does not reduce likelihood of being clinically depressed (Yonkers et al. 2011). Lack of antidepressant treatment efficacy during pregnancy is concerning given the high correlation between pre- and postpartum depression (Robertson et al. 2004), the poor pregnancy outcomes associated with depression (Grote et al. 2010), and the risk of harm associated with antidepressant medication (Bellantuono et al. 2015; Grigoriadis et al. 2013a; Palmsten et al. 2013). SSRIs have been associated with congenital heart malformations (Grigoriadis et al. 2013a) and postpartum hemorrhages (Palmsten et al. 2013), and some SNRIs have been associated with postpartum hemorrhages and hypertension (Bellantuono et al. 2015). However, with the exception of the risk of congenital heart defects associated with paroxetine (which should not be used during pregnancy [Grigoriadis et al. 2013a]), the risks associated with antidepressant use during pregnancy are small to moderate and must be weighed against the risks of depression. Notably, some antidepressants also have been found to adversely impact sleep among depressed patients in the general population, but research on their impact on sleep quality during pregnancy and postpartum is scant (Argyropoulos and Wilson 2005; Armitage 2000). During pregnancy, pharmacological treatment for depression remains fraught with challenges. To date, pregnant women and their providers lack empirical evidence to inform their decisions about the risks and benefits of antidepressant treatment.

Whereas less is known about the impact of antidepressants, associations in pregnancy and postpartum between sleep quality and depression have been empirically established (Coo et al. 2014; Giallo et al. 2016; Kamysheva et al. 2010; Skouteris et al. 2008) but with fewer studies looking at how associations change over time (Goyal et al. 2007; Wolfson et al. 2003). The majority of these studies were conducted among women without a clinical diagnosis of depression during pregnancy (Coo et al. 2014; Goyal et al. 2007; Tomfohr et al. 2015; Wolfson et al. 2003). The few studies that did include women with clinical depression did not investigate concurrent patterns of sleep and depressive symptomatology or document differences in these patterns by depression diagnosis (Field et al. 2007; Okun et al. 2011; Ruiz-Robledillo et al. 2015). Additionally, how antidepressant medications impact both sleep and the course of depressive symptomatology for pregnant and postpartum women remains unclear. Okun et al. (2011) conducted the only study, to our knowledge, on sleep and depression during pregnancy that included measures of antidepressant use. In a subgroup of pregnant women without a current depression diagnosis, the study documented associations between sleep quality and depressive symptomatology when controlling for the impact of antidepressants (Okun et al. 2011). 
By assessing antidepressant use across time, allowing use to vary over time as it does in the natural world, the current study fills a gap in the literature as there are few pregnancy or postpartum longitudinal studies of time-varying medication use (Munk-Olsen et al. 2012; Ververs et al. 2006). Furthermore, a lack of longitudinal designs and comparison groups significantly limits the conclusions that can be drawn from the existing literature. Our study expands upon prior work by specifically exploring the moderating effects of antidepressants on subjective sleep in a sample of clinically depressed and non-depressed women from pregnancy through 6-month postpartum. Empirically derived associations among antidepressant use, sleep, and depressive symptoms in both currently depressed and remitted women support the need to investigate the interactions among these factors over time in women with and without a depression diagnosis during pregnancy. Understanding how these factors influence each other during pregnancy and postpartum is key to discovering existing barriers to symptom management and improving both treatment for depression during pregnancy and prevention of postpartum depression.

The aims of this study were (a) to examine the role of antidepressant use on women's sleep quality during pregnancy through 6-month postpartum among women with and without a depressive disorder during pregnancy and (b) to assess the moderating impact of sleep disturbance and antidepressant use on ongoing patterns of depression severity during pregnancy through 6-month postpartum for women with and without a depressive disorder during pregnancy. We hypothesized that sleep disturbance would be worse among women using antidepressants. We also hypothesized that sleep disturbance in the presence of antidepressant use would lead to increased depression severity at later time points.

\section{Method}

\section{Participants}

The current study is a secondary analysis of data from a larger prospective, naturalistic longitudinal cohort study of Selective Serotonin Reuptake Inhibitor (SSRI) use during pregnancy and the effects on fetal, infant, and maternal outcomes during pregnancy through 6-month postpartum. Participants were self-referred from community obstetric offices between 2008 and 2012. Potential participants were phone-screened for eligibility and invited to participate if they met the following criteria: were between the ages of 18-40, spoke English or Spanish; were 23-34-weeks gestation with a healthy, singleton pregnancy; reported no prenatal illicit substance use, medication-dependent hypertension or diabetes; reported less than $1 / 2$ drink of alcohol/day, less than 5 cigarettes/day in the first trimester and no smoking in second or third trimesters. Current non-use of illicit substances and nonsmoking status were confirmed via urine sample with a 10-drug screening panel and a nicotine/cotinine strip (DOA-10, DOA-NIC; Craig Medical). Participants must have received routine prenatal care including a diagnostic ultrasound to rule out fetal abnormalities prior to study enrollment. Of the 465 potential participants screened for the parent study, 243 met parent study eligibility criteria. Of these 243 participants, 28 were excluded from the current analyses for one or more of the following reasons: delivered their infants at $<36$ weeks gestational age, did not provide data at least once after delivery, or were diagnosed with an Axis I psychiatric disorder other than a unipolar mood disorder. The parent study was 
approved by the Women \& Infants Hospital of Rhode Island Institutional Review Board. Informed consent was obtained from all individual participants included in the study. Assessments occurred at The Brown Center for the Study of Children at Risk, a research center at Women and Infants Hospital in Providence, RI.

\section{Measures}

Depression group-The Structured Clinical Interview for DSM-IV-TR Axis I DisordersNon-Patient Edition (SCID-I/NP; First et al. 2002) was administered at either the trimester 2 or the trimester 3 study visit and used to determine current and lifetime psychiatric diagnoses according to the Diagnostic and Statistical Manual of Mental Disorders Fourth Edition (DSM-IV-TR; American Psychological Association 2000). Participants were categorized as having a depressive disorder during pregnancy if they met diagnostic criteria on the SCID for a unipolar mood disorder (i.e., major depressive disorder, minor depressive disorder, dysthymia or depressive disorder not otherwise specified) at any point during pregnancy. Participants who did not meet criteria for any type of depressive disorder during pregnancy were categorized as without depressive disorder during pregnancy.

Antidepressant use-The Timeline Follow Back (TLFB) survey (Sobell et al. 1996) was used to assess participants' use of and compliance with antidepressants, nonpharmacological treatment for depression, other prescribed medications, nicotine, alcohol, and other substances. The TLFB provides reliable measurement of medication adherence in clinical trials (Ingersoll et al. 2011). At each study visit, the TLFB was used to ask about daily medication and other substance use, frequency, dosage, and compliance. The TLFB assessed antidepressant use (yes $V$. no) at each time point. Observations in which participants used benzodiazepines or hypnotic medication were excluded from the analyses (75 observations [8.2\%], <20 at each time point) to statistically control for variance introduced by these extraneous medications.

Time-Time points trimester 2, trimester 3, and postpartum months 1 and 6 were examined as the within-subjects effect.

Sleep quality-The Pittsburgh Sleep Quality Index (PSQI) was used to measure global sleep quality (Buysse et al. 1989). The PSQI is a reliable, valid, and standardized self-report measure of sleep quality assessed retrospectively over a 1-month period (Buysse et al. 1989; Mollayeva et al. 2016). Higher scores reflect worse sleep quality.

Depression severity-The Inventory of Depressive Symptomatology-Clinician Rated (IDS-C) was used to measure depression severity (Rush et al. 1996). The IDS-C is a 30-item semi-structured interview that assesses the severity of depressive symptoms for the week prior to the interview. The IDS-C is reliable, valid, and sensitive to change in depressive symptoms (Trivedi et al. 2004).

Demographic variables-Basic demographic information was collected by interview and self-report forms. The Hollingshead Index of Socioeconomic Status (SES) characterized participants' SES; categories 4 and 5 were rated as low SES (Gottfried 1985). Body mass 
index (BMI) was calculated using maternal report of pre-pregnancy weight and height measured at study entry.

\section{Covariates}

Candidate covariates comprised the following possible confounding variables: number of prenatal care visits, number of pregnancies, number of living children, BMI, SES, race, and marital status. Criteria for covariate selection were as follows: (a) differed significantly between depression groups, (b) were associated with depression severity on the IDS-C at $p$ $\leq .2$, and (c) were not highly correlated with other covariates (i.e., $r<.7$ ).

\section{Statistical analyses}

Participant characteristics were analyzed by depression group and antidepressant use during pregnancy. Univariate analyses of variance were used to test mean differences for the continuous variables whereas generalized loglinear models were used for categorical variables.

Associations between sleep quality, depression severity and antidepressant use were examined with two separate linear mixed models. In the first mixed-model, the outcome variable was sleep quality (PSQI) and the explanatory variables were antidepressant use (yes v. no; a time-varying variable measured at each assessment time point), depression group (no depressive disorder during pregnancy v. depressive disorder during pregnancy), and time (trimester $2 \mathrm{v}$. trimester $3 \mathrm{v}$. postpartum month $1 \mathrm{v}$. postpartum month 6). The model included two covariates: SES and number of pregnancies. In the second mixed-model, the outcome variable was depression severity (IDS-C) and the explanatory variables were antidepressant use, depression group, sleep quality, and time. The second mixed model included the covariates SES, number of pregnancies, and BMI.

Depression group remained a static dichotomous variable in the models tested. Sleep quality and antidepressant use were allowed to vary over time to account for change over time (Singer and Willett 2003; See Fig. 1). Post hoc testing was conducted for significant secondorder interaction effects and any first-order interaction effects not included as part of a higher-order interaction. These post hoc tests of the second-order interaction effects consisted of simple first-order interaction testing followed by simple main effects testing of significant first-order interactions. All tests were adjusted for multiple comparisons using the Benjamini-Hochberg false discovery rate approach (Benjamini and Hochberg 1995). Analyses were conducted using SPSS 21.0 software (IBM, Somers, N.Y.).

\section{Results}

\section{Participants}

The 28 participants excluded from study analyses had significantly higher IDS-C scores (28.08 [SD $=17.98]$ v. 18.34 [11.18], $F[1,73]=6.46, p=.013)$ and PSQI scores $(10.82$ [4.70] v. 8.24 [4.72], $F[1,161]=5.70, p=.018)$ than the 215 who were included. Excluded participants also had a greater number of pregnancies $(3.32$ [2.92] v. $2.21[1.27], F[1,238]=$ 
$12.76, p<.001)$ and number of living children (1.32 [SD = 1.63] v.74 [.87], $F[1,238]=8.7$, $p<.004)$.

Among the 215 participants included in the study, all women (100\%) who did not have a depressive disorder during pregnancy and used antidepressants during pregnancy had a history of a prior depressive disorder but remained in remission throughout the pregnancy. For women who did not have a depressive disorder during pregnancy and who did not use antidepressants, $8(10 \%)$ of the 78 had a history of a prior depressive disorder. One hundred ten women (51\%) completed all 4 assessments; 58 (27\%) completed 2-3 assessments; and $47(22 \%)$ completed 1 assessment. Table 1 presents the demographic characteristics by total sample $(\mathrm{N}=215)$ and by depression group (No depressive disorder during pregnancy $[N=$ 104] $v$. Depressive disorder during pregnancy $[N=111])$. Within-group analyses showed that, compared to women not using antidepressants, women using antidepressants (both with and without a depressive disorder), were older, less likely to be Hispanic and more likely to be married. Between-group analyses showed that, compared to women without a depressive disorder during pregnancy, women with a depressive disorder during pregnancy had a higher number of pregnancies, more living children, greater BMI, and were more likely to be of minority status and unmarried.

\section{Subjective sleep quality}

Table 2 presents the mixed model results for the analysis of sleep quality, which resulted in two significant first-order interactions: Depression Group X Antidepressant Use, and Depression Group X Time. Fig. 2 illustrates sleep quality scores over time by concurrent use of antidepressant medications within Depression Group. Regarding the Depression Group X Antidepressant Use interaction, for participants without a depressive disorder during pregnancy, those with antidepressant use had worse sleep quality overall than those without antidepressant use (mean difference $=2.04[S E=.6], p<.001$ ). Among participants with a depressive disorder during pregnancy, the sleep quality of those with antidepressant use and without antidepressant use was not significantly different. Regarding the Depression Group $\mathrm{X}$ Time interaction, for participants without a depressive disorder during pregnancy, sleep quality was significantly better in postpartum month 6 compared to all other measurement points $(p s<.05)$ whereas participants with a depressive disorder during pregnancy had significantly improved scores in postpartum month 6 only compared to trimester 3 , which was the worst score compared to all other measurement points ( $p s<.05)$.

\section{Depression severity}

Table 3 presents the mixed model results for the analysis of depression severity. There were two significant second-order interactions: Antidepressant Use X Depression Group X Sleep and Antidepressant Use X Depression Group X Time.

Regression coefficients for the Antidepressant Use X Depression Group X Sleep interaction are presented in Fig. 3. Post hoc analyses indicated that, among women with a depressive disorder during pregnancy, there was a significant Antidepressant Use X Sleep interaction. The magnitude of the effect of sleep quality on depression severity was significantly larger for women using antidepressants than for women not using antidepressants. Among women 
with a depressive disorder during pregnancy and with antidepressant use, for every 1-point increase on the PSQI, depression severity on the IDS-C increased by $2.65(\mathrm{SE}=.28)$ points ( $p s<.05$; see Fig. 3). Among women without a depressive disorder during pregnancy, there was no significant difference in the effect of sleep quality on depression severity by antidepressant use.

For the Antidepressant Use X Depression Group X Time interaction, Fig. 4 depicts the change in depression severity scores over time by antidepressant use for women with and without a depressive disorder during pregnancy. Post hoc analyses indicated that, among participants with a depressive disorder during pregnancy, antidepressant use moderated depression severity over time. Participants with a depressive disorder during pregnancy using antidepressants had significantly higher depression severity than their non-medicated counterparts at postpartum month 6 (mean difference $=8.48$ [2.31], $p<.001$ ). Participants with a depressive disorder during pregnancy who were not using antidepressants had their highest depression severity at trimester 3 ( $p s<.01$; in the clinically moderate range) and their lowest depression severity at postpartum month 6 ( $p$ s $<.001$; in the non-clinical range) whereas there was no significant change over time in depression severity for participants using antidepressants. The depression severity for participants using antidepressants remained in the clinically significant mild to moderate range throughout the study period. Among participants without a depressive disorder during pregnancy, there were no significant differences in depression severity associated with time or antidepressant use; the depression severity of these women started in the nonclinical range and remained low throughout the study period.

To account for the potential influence of change in medication status over time on differences in level of depression severity at postpartum month 6 between those using and not using antidepressants, planned comparisons were conducted of change over time in medication use within depression groups for participants completing all three assessments $(N=141)$. There were no significant differences within depression groups for the percentage of participants using antidepressant medication from pregnancy to either postpartum assessment. Additionally, depression severity scores for women with a depressive disorder during pregnancy who began taking antidepressant medication in postpartum month 1 were not significantly different from women with a depressive disorder during pregnancy who did not take medication in postpartum month 1 (Mean $=23.85$ [SE = 3.48]; 20.06 [2.12]) or in postpartum month 6 (17.54 [3.58]; 20.05 [2.12]).

Consistent with an approach used in previous studies (Roane et al. 2013; Skouteris et al. 2008), the mixed model analysis was re-run with the IDS-C as the outcome variable with sleep questions removed in order to assess whether or not the effect of the PSQI on the IDS$\mathrm{C}$ was inflated by this sleep question. The mixed model was also re-run with the anhedonia question from the PSQI removed. In both cases, the same interaction terms remained significant. 


\section{Discussion}

This prospective study is the first to our knowledge to examine the moderating effects of time-varying antidepressant use on depression severity and subjective sleep quality from pregnancy to postpartum in women with and without a depressive disorder during pregnancy. For women with a depressive disorder in pregnancy, antidepressant use did not affect sleep quality but did affect the trajectory of depression severity. Without antidepressant use, depression severity worsened in trimester 3 but improved by 6-month postpartum. Conversely, with antidepressant use, depression severity remained fairly stable over time, so that by postpartum month 6 , women who were using antidepressants were significantly more depressed than their non-medicated counterparts. There are factors, dynamic in pregnancy and postpartum, that may impact depression severity differently for women with and without antidepressant use. Specific examples of dynamic factors include low back pain and leg cramps, in addition to restless sleep and stressful dreams, all of which have been shown to worsen in trimester 3 relative to other trimesters (Bei et al. 2015a; Lee 1998). Previous studies have documented a negative effect of physiological changes in late pregnancy on sleep quality and mood (e.g., Kamysheva et al. 2010), and these physiological changes may be more disruptive or noticeable for depressed women. The time effects of the current study point to the rapidly changing physiological framework across which pregnancy and postpartum antidepressant treatment spans and highlight the unremitting nature of depression within the context of antidepressant treatment and untreated sleep disturbance.

The pattern of results was different for women who did not have a depressive disorder during pregnancy; women who were taking antidepressants reported worse sleep quality than their non-medicated counterparts—on par with the level of sleep disturbance in women with a depressive disorder during pregnancy. This effect was stable over time and was not accounted for by depression severity. Similarly, uncorrected analyses in the 2011 study by Okun and colleagues showed more difficulty falling and staying asleep in women with remitted depression treated with antidepressants compared with controls; however, the effect was lost after corrections and measures of sleep quality were not included. Subjective sleep disturbance in women with remitted depression who are taking antidepressants may be a unique effect of antidepressants or a residual symptom of remitted depression, or both. In non-pregnant samples, antidepressants, particularly SSRIs, have been shown to prolong sleep onset latency (Vasar et al. 1994), increase middle-of-the-night wakefulness, reduce deep sleep (Armitage 2000; Oberndorfer et al. 2000), reduce total sleep time (Sharpley et al. 1996), and lead to changes in sleep architecture, such as REM suppression (Argyropoulos and Wilson 2005). Previous studies in non-pregnant samples have also documented sleep disturbance as a residual symptom of remitted depression (Iovieno et al. 2011; Nierenberg et al. 2010). Nierenberg et al. (2010) found that $71 \%$ of participants with remitted depression reported sleep disruption following 1 year of maintenance therapy with antidepressants. Studies using objective measures of sleep have also found that disruptions in sleep architecture, such as reduced REM sleep latency and a decreased delta sleep ratio, can persist following remission (Lee et al. 1993).

In addition to moderating level of sleep disturbance, antidepressant use moderated the strength of the effect of sleep disturbance on depression severity. At every time point, the 
effect of sleep quality on depression severity was most pronounced among prenatally depressed women who were using antidepressants compared with non-medicated women whether or not they were prenatally depressed. Importantly, among women with a depressive disorder during pregnancy, levels of depression severity for women using and not using antidepressants were similar during pregnancy and the first month postpartum. Thus, this strengthened relationship between sleep quality and depression severity among these women was independent of level of depression severity. These findings support a cumulative effect of exposure to depression and antidepressants on the degree to which sleep quality and depression severity exacerbate each other.

Despite the established link between depression severity and subjective sleep quality (Field et al. 2007; Goyal et al. 2007; Wolfson et al. 2003), less is known about the effect of antidepressant use on how sleep impacts depression and vice versa. Okun et al. (2011) found that during trimester 2 of pregnancy women with both depression and antidepressant use experienced more difficulty initiating sleep and napped more than their non-depressed counterparts. The Okun study did not observe these women postpartum, but in the present study, this group of women - those who had a depressive disorder during pregnancy and antidepressant use-did not experience the reduction in depression severity by postpartum month 6 experienced by their non-medicated counterparts. The possibility that this effect was due to women starting on antidepressants after the first postpartum month because of increased depression severity was addressed with secondary analyses, which found no significant difference from one time point to the next in the number of women within depression groups who changed antidepressant medication status. Furthermore, there was no significant difference in depression severity at 1- and 6-month postpartum in women with a depressive disorder who started an antidepressant postpartum, compared with those who continued to abstain from medication postpartum. Therefore, it seems that selection bias did not account for the difference in depression severity at postpartum month 6.

In sum, this study found clinically disturbed sleep from pregnancy to postpartum in women with antidepressant use and/or prenatal depression and found that the sleep disturbance in these women was significantly associated with their depression severity over time.

Furthermore, in women with prenatal depression, antidepressant use continued to be significantly associated with clinically elevated depression severity as far out as 6-month postpartum. This culmination of findings suggests poor sleep as a risk factor for the course of depression, particularly for women with antidepressant use. The findings merit more highly prioritizing investigations of sleep interventions as a target for lowering depression severity in pregnant women with current depression and sleep disturbance in both actively depressed and remitted women. The elevated sleep disturbance documented in this study among antidepressantusing women with and without depression suggests a potential need for a combined treatment for sleep disturbance and depression or, at the least, a form of depression treatment that more adequately addresses the symptom of sleep disturbance.

\section{Limitations}

Previous studies have documented associations among some combination of sleep and depression variables but were limited by a shorter timeframe or fewer time points, by 
unaddressed potential confounds such as antidepressant use, and by a lack of comparison groups. Our investigation improved upon existing sleep and depression research in pregnant and postpartum samples by modeling interaction effects of antidepressant use, sleep quality, and time on depression severity in prenatally depressed, remitted, and non-depressed women throughout pregnancy and postpartum and by allowing antidepressant use to vary over time.

Limitations include potential selection as well as reporting bias. Because antidepressant use naturally varied over time, depression severity and sleep disturbance may be acting on antidepressant use as well as the converse. Furthermore, generalizability of study results to severely depressed women is limited; the 28 women excluded from the study (due to not completing at least one assessment or being diagnosed with an Axis 1 disorder other than unipolar depression) were more depressed than study participants.

This study used a subjective self-report sleep measure, the PSQI, as the measure of sleep quality. Overlapping symptom measurement is an inherent confound in any study of depression; however, this study limited potential confounding between the PSQI and the IDS-C by examining the models using both the full versions and modified versions of both the IDS-C and the PSQI. The modified IDS-C eliminated sleep-related questions whereas the modified PSQI eliminated an item (i.e., decreased enthusiasm) possibly overlapping with depression. There were no differences in outcomes with the modified scales.

Hypnotic medication (e.g., trazadone, zolpidem) use as a potential confounding factor was controlled by excluding time points from analysis in which participants used hypnotics; however it was beyond the scope of this study to examine the influence of hypnotics on depression severity. Evidence on the benefits of sleep treatments (behavioral and pharmacological) on depression is substantial (Bei et al. 2015b; Taylor and Pruiksma 2014) and worthy of further investigation in pregnant and postpartum samples (Khazaie et al. 2013; Swanson et al. 2013).

Whether differences existed between specific medication types or dosages reported was not addressed in the current investigation. Furthermore, we did not examine the role of nonpharmacological treatments (e.g., psychotherapy) on sleep quality or depression severity, which is another area of needed study. Although the challenge of dissecting causal relationships between sleep, depression, and antidepressant use remains, the present study is the first to examine the course of these relationships, an important step in accurately understanding the utility of antidepressants in treating pregnancy and postpartum depression.

\section{Conclusions}

This study highlights sleep disturbance as an insidious symptom of depression not adequately addressed by the presence of antidepressants. Women with remitted depression in pregnancy who used antidepressant medications continued to have clinical levels of sleep disturbance. The ongoing use of antidepressant medication may be associated with ongoing sleep disturbance and may represent risk for depression reoccurrence. For women with clinical depression in pregnancy, the unique effect of sleep disturbance on depression severity was particularly strong and remained strong over time. Therefore, for both women 
with remitted and women with active depression during pregnancy, greater attention to improving sleep quality could be a key factor in improving treatment outcomes. Studies in the general population have documented behavioral and pharmacological sleep treatments improving both sleep and depression (Bei et al. 2015b; Taylor et al. 2007; Taylor and Pruiksma 2014) and a few studies have also documented this effect in pregnant and postpartum women (Khazaie et al. 2013; Swanson et al. 2013). Additionally, several studies in non-pregnant samples investigating supplementing antidepressants with sleep interventions (both pharmacological and behavioral) in women with depression and cooccurring sleep disturbance found reduced depression severity and improved sleep quality compared with antidepressants alone (Fava et al. 2006; Manber et al. 2008). Sleep education, assessment, and treatment for pregnant patients being treated with antidepressants before trimester 3 may improve outcomes for these women and their infants. Future investigations of treatment for both depression and sleep disturbance, administered during pregnancy with adequate postpartum follow-ups (O'Connor et al. 2016), will help sort out the effects of antidepressant use, which will aid in the continued development of safe treatments for sleep and depression during this critical time with potential for intergenerational benefit.

\section{Acknowledgments}

Funding This study was funded by The National Institutes of Health, grant R01MH078033 (PI: Amy Salisbury, $\mathrm{PhD})$.

\section{References}

Argyropoulos SV, Wilson SJ (2005) Sleep disturbances in depression and the effects of antidepressants. Int Rev Psychiatry 17:237-245. doi: 10.1080/09540260500104458 [PubMed: 16194795]

Armitage R (2000) The effects of antidepressants on sleep in patients with depression. Can J Psychiatr 45:803-809. doi:10.1177/070674370004500903

American Psychological Association (2000) Diagnostic and statistical manual of mental disorders, fourth edition, text revision. American Psychiatric Publishing, Washington

Battle CL, Zlotnick C, Miller IW, Pearlstein T, Howard M (2006) Clinical characteristics of perinatal psychiatric patients: a chart review study. J Nerv Ment Dis 194:369-377. doi:10.1097/01.nmd. 0000217833.49686.c0 [PubMed: 16699387]

Bei B, Coo S, Trinder J (2015a) Sleep and mood during pregnancy and the postpartum period. Sleep Medicine Clinics 10:25-33. doi:10.1016/j.jsmc.2014.11.011 [PubMed: 26055670]

Bei B, Ong JC, Rajaratnam SM, Manber R (2015b) Chronotype and improved sleep efficiency independently predict depressive symptom reduction after group cognitive behavioral therapy for insomnia. J Clin Sleep Med 11:1021-1027. doi:10.5664/jcsm.5018 [PubMed: 25845891]

Bellantuono C, Vargas M, Mandarelli G, Nardi B, Martini MG (2015) The safety of serotoninnoradrenaline reuptake inhibitors (SNRIs) in pregnancy and breastfeeding: a comprehensive review. Hum Psychopharmacol 30:143-151. doi:10.1002/hup.2473 [PubMed: 25784291]

Benjamini Y, Hochberg Y (1995) Controlling the false discovery rate: a practical and powerful approach to multiple testing. J R Stat Soc Series B Stat Methodol 57:289-300

Buysse DJ, Reynolds CF 3rd, Monk TH, Berman SR, Kupfer DJ (1989) The pittsburgh sleep quality index: a new instrument for psychiatric practice and research. Psychiatry Res 28:193-213 [PubMed: 2748771]

Coo S, Milgrom J, Trinder J (2014) Mood and objective and subjective measures of sleep during late pregnancy and the postpartum period. Behav Sleep Med 12:317-330. doi: 10.1080/15402002.2013.801348 [PubMed: 24128191] 
Cooper WO, Willy ME, Pont SJ, Ray WA (2007) Increasing use of antidepressants in pregnancy. Am J Obstet Gynecol 196(544): e541-e545. doi:10.1016/j.ajog.2007.01.033

Davidson JR (2010) Major depressive disorder treatment guidelines in America and Europe. J Clin Psychiatry 71(Suppl E1):e04. doi:10.4088/JCP.9058se1c.04gry

Fava M, McCall WV, Krystal A, Wessel T, Rubens R, Caron J, Amato D, Roth T (2006) Eszopiclone co-administered with fluoxetine in patients with insomnia coexisting with major depressive disorder. Biol Psychiatry 59:1052-1060. doi:10.1016/j.biopsych.2006.01.016 [PubMed: 16581036]

Field T, Diego M, Hernandez-Reif M (2006) Prenatal depression effects on the fetus and newborn: a review. Infant Behav Dev 29:445-455. doi: 10.1016/j.infbeh.2006.03.003 [PubMed: 17138297]

Field T, Diego M, Hernandez-Reif M, Figueiredo B, Schanberg S, Kuhn C (2007) Sleep disturbances in depressed pregnant women and their newborns. Infant Behav Dev 30:127-133. doi:10.1016/ j.infbeh.2006.08.002 [PubMed: 17292785]

First M, Spitzer R, Gibbon M (2002) Structured clinical interview for DSM-IV-TR axis I disorders, research version, non-patient edition (SCID-I/NP) Biometrics Research, New York State Psychiatric Institute, New York

Gavin NI, Gaynes BN, Lohr KN, Meltzer-Brody S, Gartlehner G, Swinson T (2005) Perinatal depression: a systematic review of prevalence and incidence. Obstet Gynecol 106:1071-1083. doi: 10.1097/01.AOG.0000183597.31630.db [PubMed: 16260528]

Giallo R, Gartland D, Woolhouse H, Brown S (2016) "I didn't know it was possible to feel that tired": exploring the complex bidirectional associations between maternal depressive symptoms and fatigue in a prospective pregnancy cohort study. Arch Womens Ment Health 19: 25-34. doi: 10.1007/s00737-014-0494-8 [PubMed: 25577337]

Gottfried A (1985) Measures of socioeconomic status in child development research: data and recommendations. Merrill Palmer Q 31:85-92

Goyal D, Gay CL, Lee KA (2007) Patterns of sleep disruption and depressive symptoms in new mothers. J Perinat Neonatal Nurs 21: 123-129. doi:10.1097/01.JPN.0000270629.58746.96 [PubMed: 17505232]

Grigoriadis S, VonderPorten EH, Mamisashvili L, Roerecke M, Rehm J, Dennis CL, Koren G, Steiner M, Mousmanis P, Cheung A, Ross LE (2013a) Antidepressant exposure during pregnancy and congenital malformations: is there an association? A systematic review and meta-analysis of the best evidence. J Clin Psychiatry 74:e293-e308. doi:10.4088/JCP.12r07966 [PubMed: 23656855]

Grigoriadis S, VonderPorten EH, Mamisashvili L, Tomlinson G, Dennis CL, Koren G, Steiner M, Mousmanis P, Cheung A, Radford K, Martinovic J, Ross LE (2013b) The impact of maternal depression during pregnancy on perinatal outcomes: a systematic review and meta-analysis. J Clin Psychiatry 74:e321-e341. doi:10.4088/JCP.12r07968 [PubMed: 23656857]

Grote NK, Bridge JA, Gavin AR, Melville JL, Iyengar S, Katon WJ (2010) A meta-analysis of depression during pregnancy and the risk of preterm birth, low birth weight, and intrauterine growth restriction. Arch Gen Psychiatry 67:1012-1024. doi:10.1001/archgenpsychiatry.2010.111 [PubMed: 20921117]

Ingersoll KS, Farrell-Carnahan L, Cohen-Filipic J, Heckman CJ, Ceperich SD, Hettema J, MarzaniNissen G (2011) A pilot randomized clinical trial of two medication adherence and drug use interventions for HIV+ crack cocaine users. Drug Alcohol Depen 116: 177-187. doi:10.1016/ j.drugalcdep.2010.12.016

Iovieno N, van Nieuwenhuizen A, Clain A, Baer L, Nierenberg AA (2011) Residual symptoms after remission of major depressive disorder with fluoxetine and risk of relapse. Depress Anxiety 28:137-144. doi:10.1002/da.20768 [PubMed: 21284066]

Kamysheva E, Skouteris H, Wertheim EH, Paxton SJ, Milgrom J (2010) A prospective investigation of the relationships among sleep quality, physical symptoms, and depressive symptoms during pregnancy. J Affect Disord 123:317-320. doi:10.1016/j.jad.2009.09.015 [PubMed: 19822370]

Khazaie H, Ghadami MR, Knight DC, Emamian F, Tahmasian M (2013) Insomnia treatment in the third trimester of pregnancy reduces postpartum depression symptoms: a randomized clinical trial. Psychiatry Res 210:901-905. doi:10.1016/j.psychres.2013.08.017 [PubMed: 23993464] 
Lee JH, Reynolds CF 3rd, Hoch CC, Buysse DJ, Mazumdar S, George CJ, Kupfer DJ (1993) Electroencephalographic sleep in recently remitted, elderly depressed patients in double-blind placebo-maintenance therapy. Neuropsychopharmacology 8:143-150. doi:10.1016/ S1087-0792(98)90010-7 [PubMed: 8471126]

Lee KA (1998) Alterations in sleep during pregnancy and postpartum: a review of 30 years of research. Sleep Med Rev 2:231-242. doi:10.1016/S1087-0792(98)90010-7 [PubMed: 15310494]

Manber R, Edinger JD, Gress JL, San Pedro-Salcedo MG, Kuo TF, Kalista T (2008) Cognitive behavioral therapy for insomnia enhances depression outcome in patients with comorbid major depresssive disorder and insomnia. Sleep 31:489-495 [PubMed: 18457236]

Micheli K, Komninos I, Bagkeris E, Roumeliotaki T, Koutis A, Kogevinas M, Chatzi L (2011) Sleep patterns in late pregnancy and risk of preterm birth and fetal growth restriction. Epidemiology 22:738-744. doi:10.1097/EDE.0b013e31822546fd [PubMed: 21734587]

Mollayeva T, Thurairajah P, Burton K, Mollayeva S, Shapiro CM, Colantonio A (2016) The pittsburgh sleep quality index as a screening tool for sleep dysfunction in clinical and non-clinical samples: a systematic review and meta-analysis. Sleep Med Rev 25:52-73. doi: 10.1016/j.smrv.2015.01.009 [PubMed: 26163057]

Munk-Olsen T, Gasse C, Laursen TM (2012) Prevalence of antidepressant use and contacts with psychiatrists and psychologists in pregnant and postpartum women. Acta Psychiatr Scand 125:318-324. doi:10.1111/j.1600-0447.2011.01784.x [PubMed: 22118213]

Nierenberg AA, Husain MM, Trivedi MH, Fava M, Warden D, Wisniewski SR, Miyahara S, Rush AJ (2010) Residual symptoms after remission of major depressive disorder with citalopram and risk of relapse: a STAR*D report. Psychol Med 40:41-50. doi:10.1017/S0033291709006011 [PubMed: 19460188]

O’Connor E, Rossom RC, Henninger M, Groom HC, Burda BU (2016) Primary care screening for and treatment of depression in pregnant and postpartum women: evidence report and systematic review for the US preventive services task force. JAMA 315:388-406. doi:10.1001/jama.2015.18948 [PubMed: 26813212]

Oberndorfer S, Saletu-Zyhlarz G, Saletu B (2000) Effects of selective serotonin reuptake inhibitors on objective and subjective sleep quality. Neuropsychobiology 42:69-81 doi:26676 [PubMed: 10940762]

Okun ML, Hanusa BH, Hall M, Wisner KL (2009a) Sleep complaints in late pregnancy and the recurrence of postpartum depression. Behav Sleep Med 7:106-117. doi: 10.1080/15402000902762394 [PubMed: 19330583]

Okun ML, Kiewra K, Luther JF, Wisniewski SR, Wisner KL (2011) Sleep disturbances in depressed and nondepressed pregnant women. Depress Anxiety 28:676-685. doi:10.1002/da.20828 [PubMed: 21608086]

Okun ML, Luther JF, Wisniewski SR, Wisner KL (2013) Disturbed sleep and inflammatory cytokines in depressed and nondepressed pregnant women: an exploratory analysis of pregnancy outcomes. Psychosom Med 75:670-681. doi: 10.1097/PSY.0b013e31829cc3e7 [PubMed: 23864582]

Okun ML, Roberts JM, Marsland AL, Hall M (2009b) How disturbed sleep may be a risk factor for adverse pregnancy outcomes. Obstet Gynecol Surv 64:273-280. doi:10.1097/OGX. 0b013e318195160e [PubMed: 19296861]

Palmsten K, Hernandez-Diaz S, Huybrechts KF, Williams PL, Michels KB, Achtyes ED, Mogun H, Setoguchi S (2013) Use of antidepressants near delivery and risk of postpartum hemorrhage: cohort study of low income women in the United States. BMJ 347:f4877. doi:10.1136/bmj.f4877 [PubMed: 23965506]

Roane BM, Seifer R, Sharkey KM, Van Reen E, Bond TL, Raffray T, Carskadon MA (2013) Reliability of a scale assessing depressed mood in the context of sleep. TPM Test Psychom Methodol Appl Psychol 20:3-11. doi:10.4473/TPM20.1.1 [PubMed: 25346804]

Robertson E, Grace S, Wallington T, Stewart DE (2004) Antenatal risk factors for postpartum depression: a synthesis of recent literature. Gen Hosp Psychiatry 26:289-295. doi:10.1016/ j.genhosppsych.2004.02.006 [PubMed: 15234824] 
Ruiz-Robledillo N, Canario C, Dias CC, Moya-Albiol L, Figueiredo B (2015) Sleep during the third trimester of pregnancy: the role of depression and anxiety. Psychol Health Med 20:927-932. doi: 10.1080/13548506.2015.1017508 [PubMed: 25715641]

Rush AJ, Gullion CM, Basco MR, Jarrett RB, Trivedi MH (1996) The inventory of depressive symptomatology (IDS): psychometric properties. Psychol Med 26:477-486. doi:10.1017/ S0033291700035558 [PubMed: 8733206]

Salisbury AL, O'Grady KE, Battle CL, Wisner KL, Anderson GM, Stroud LR, Miller-Loncar CL, Young ME, Lester BM (2016) The roles of maternal depression, serotonin reuptake inhibitor treatment, and concomitant benzodiazepine use on infant neurobehavioral functioning over the first postnatal month. Am J Psychiatry 173:147-157. doi:10.1176/appi.ajp.2015.14080989 [PubMed: 26514656]

Salisbury AL, Wisner KL, Pearlstein T, Battle CL, Stroud L, Lester BM (2011) Newborn neurobehavioral patterns are differentially related to prenatal maternal major depressive disorder and serotonin reuptake inhibitor treatment. Depress Anxiety 28:1008-1019. doi:10.1002/da.20883 [PubMed: 21898709]

Sharpley AL, Williamson DJ, Attenburrow ME, Pearson G, Sargent P, Cowen PJ (1996) The effects of paroxetine and nefazodone on sleep: a placebo controlled trial. Psychopharmacology 126:50-54. doi:10.1007/BF02246410 [PubMed: 8853216]

Singer J, Willett J (2003) Applied longitudinal data analysis. Oxford University Press, New York

Skouteris H, Germano C, Wertheim EH, Paxton SJ, Milgrom J (2008) Sleep quality and depression during pregnancy: a prospective study. J Sleep Res 17:217-220. doi:10.1111/j. 1365-2869.2008.00655.x [PubMed: 18482110]

Sobell LC, Brown J, Leo GI, Sobell MB (1996) The reliability of the alcohol timeline followback when administered by telephone and by computer. Drug Alcohol Depend 42:49-54. doi: 10.1016/0376-8716(96)01263-X [PubMed: 8889403]

Swanson LM, Flynn H, Adams-Mundy JD, Armitage R, Arnedt JT (2013) An open pilot of cognitivebehavioral therapy for insomnia in women with postpartum depression. Behav Sleep Med 11:297307. doi:10.1080/15402002.2012.683902 [PubMed: 23216373]

Taylor DJ, Lichstein KL, Weinstock J, Sanford S, Temple JR (2007) A pilot study of cognitivebehavioral therapy of insomnia in people with mild depression. Behav Ther 38:49-57. doi: 10.1016/j.beth.2006.04.002 [PubMed: 17292694]

Taylor DJ, Pruiksma KE (2014) Cognitive and behavioural therapy for insomnia (CBT-I) in psychiatric populations: a systematic review. Int Rev Psychiatry 26:205-213. doi: 10.3109/09540261.2014.902808 [PubMed: 24892895]

Tomfohr LM, Buliga E, Letourneau NL, Campbell TS, Giesbrecht GF (2015) Trajectories of sleep quality and associations with mood during the perinatal period. Sleep 38:1237-1245. doi:10.5665/ sleep.4900 [PubMed: 25845691]

Trivedi MH, Rush AJ, Ibrahim HM, Carmody TJ, Biggs MM, Suppes T, Crismon ML, Shores-Wilson K, Toprac MG, Dennehy EB, Witte B, Kashner TM (2004) The inventory of depressive symptomatology, clinician rating (IDS-C) and self-report (IDS-SR), and the quick inventory of depressive symptomatology, clinician rating (QIDS-C) and self-report (QIDS-SR) in public sector patients with mood disorders: a psychometric evaluation. Psychol Med 34:73-82. doi:10.1017/ S0033291703001107 [PubMed: 14971628]

Vasar V, Appelberg B, Rimon R, Selvaratnam J (1994) The effect of fluoxetine on sleep: a longitudinal, double-blind polysomnographic study of healthy volunteers. Int Clin Psychopharmacol 9:203-206 [PubMed: 7814830]

Ververs T, Kaasenbrood H, Visser G, Schobben F, de Jong-van den Berg L, Egberts T(2006) Prevalence and patterns of antidepressant drug use during pregnancy. Eur J Clin Pharmacol 62:863-870. doi:10.1007/s00228-006-0177-0 [PubMed: 16896784]

Wolfson AR, Crowley SJ, Anwer U, Bassett JL (2003) Changes in sleep patterns and depressive symptoms in first-time mothers: last trimester to 1-year postpartum. Behav Sleep Med 1:54-67. doi:10.1207/S15402010BSM0101_6 [PubMed: 15600137] 
Wu M, Li X, Feng B, Wu H, Qiu C, Zhang W (2014) Poor sleep quality of third-trimester pregnancy is a risk factor for postpartum depression. Med Sci Monitor 20:2740-2745. doi:10.12659/MSM. 891222

Yonkers KA, Gotman N, Smith MV, Forray A, Belanger K, Brunetto WL, Lin H, Burkman RT, Zelop CM, Lockwood CJ (2011) Does antidepressant use attenuate the risk of a major depressive episode in pregnancy? Epidemiology 22:848-854. doi:10.1097/EDE.0b013e3182306847 [PubMed: 21900825] 


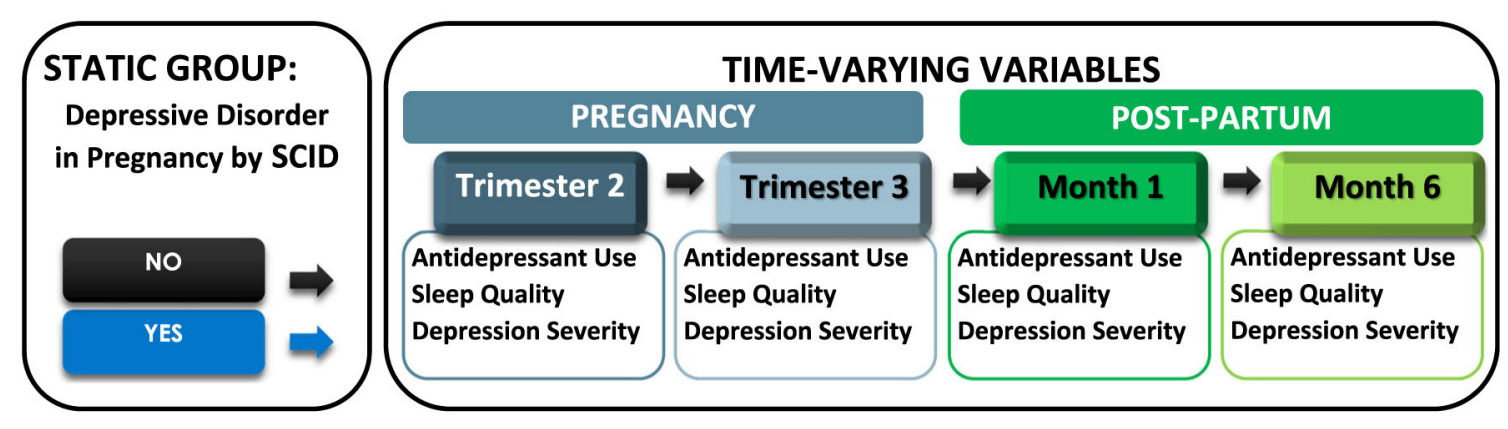

Fig. 1.

Schematic of study design. Women were classified as non-depressed or depressed during the current pregnancy according to the SCID. Antidepressant use, sleep quality, and depression severity were measured for each group at Trimester 2, Trimester 3, 1-month postpartum, and 6-month postpartum 
a. No Depressive Disorder in Pregnancy

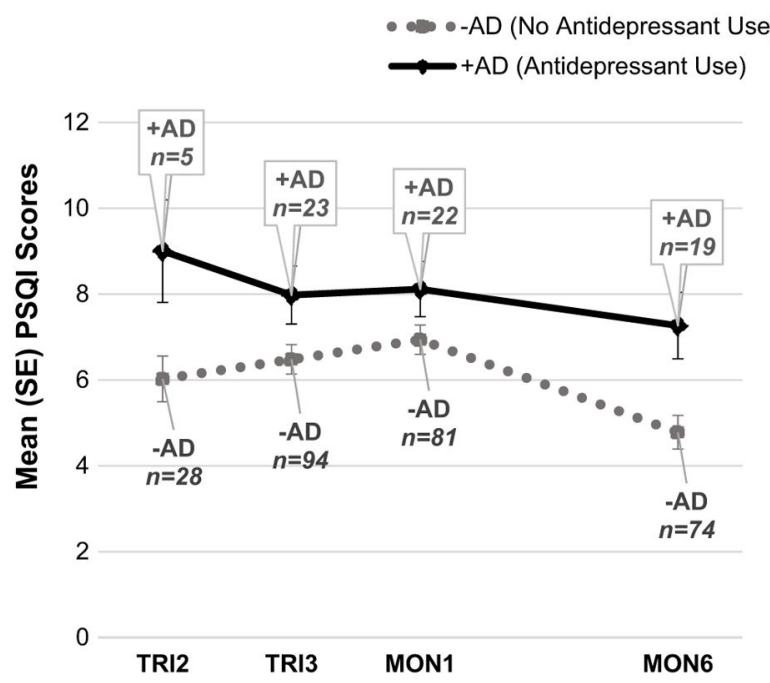

b. Depressive Disorder in Pregnancy

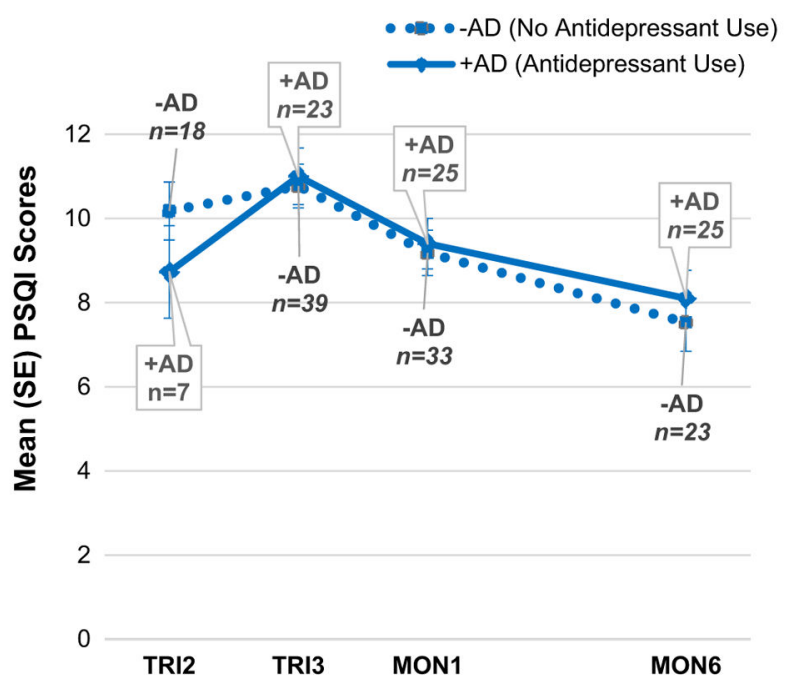

Fig. 2.

Pittsburgh Sleep Quality Index (PSQI) scores measured during Trimesters 2 and 3 in pregnancy, 1- and 6-month postpartum. a PSQI scores by concurrent antidepressant use for women without a depressive disorder during pregnancy. b PSQI scores by concurrent antidepressant use for women with a depressive disorder during pregnancy. Higher scores indicate worse sleep quality 
No Depressive Disorder during Pregnancy

Depressive Disorder during Pregnancy

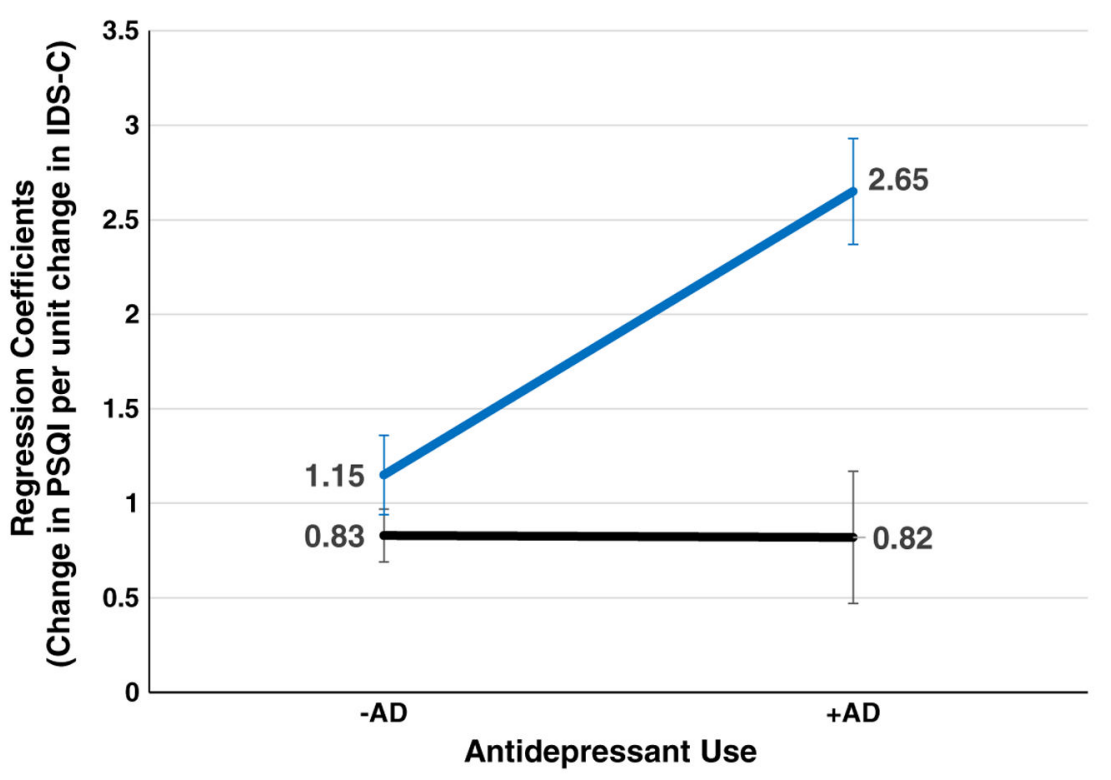

Fig. 3.

Regression coefficients for the change in Inventory of Depressive Symptomatology (IDS-C) scores per unit change in Pittsburgh Sleep Quality Index (PSQI) by concurrent antidepressant use for women with and without a depressive disorder during pregnancy 
a. No Depressive Disorder during Pregnancy

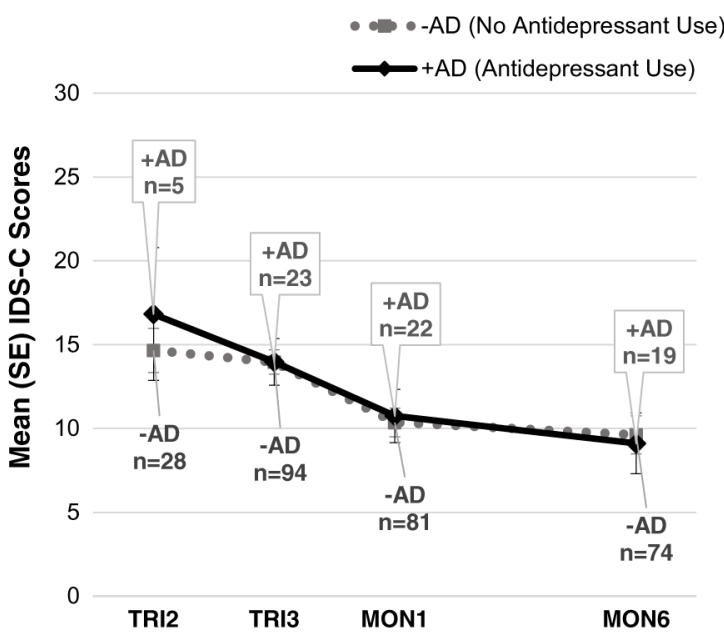

b. Depressive Disorder during Pregnancy

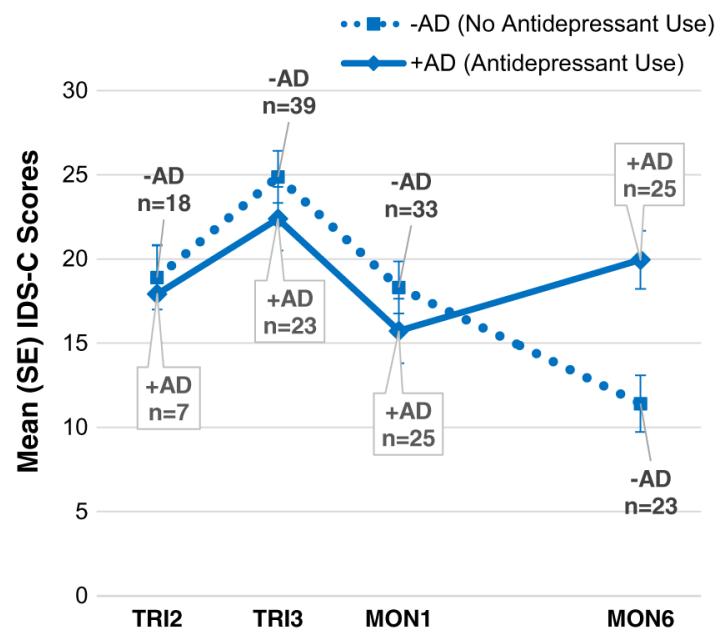

Fig. 4.

Inventory of Depressive Symptomatology (IDS-C) scores measured during Trimesters 2 and 3 in pregnancy, 1- and 6-month postpartum. a IDS-C scores by concurrent antidepressant use for women without a depressive disorder during pregnancy. b IDS-C scores by concurrent antidepressant use for women with a depressive disorder during pregnancy. Higher scores indicate higher depression severity 


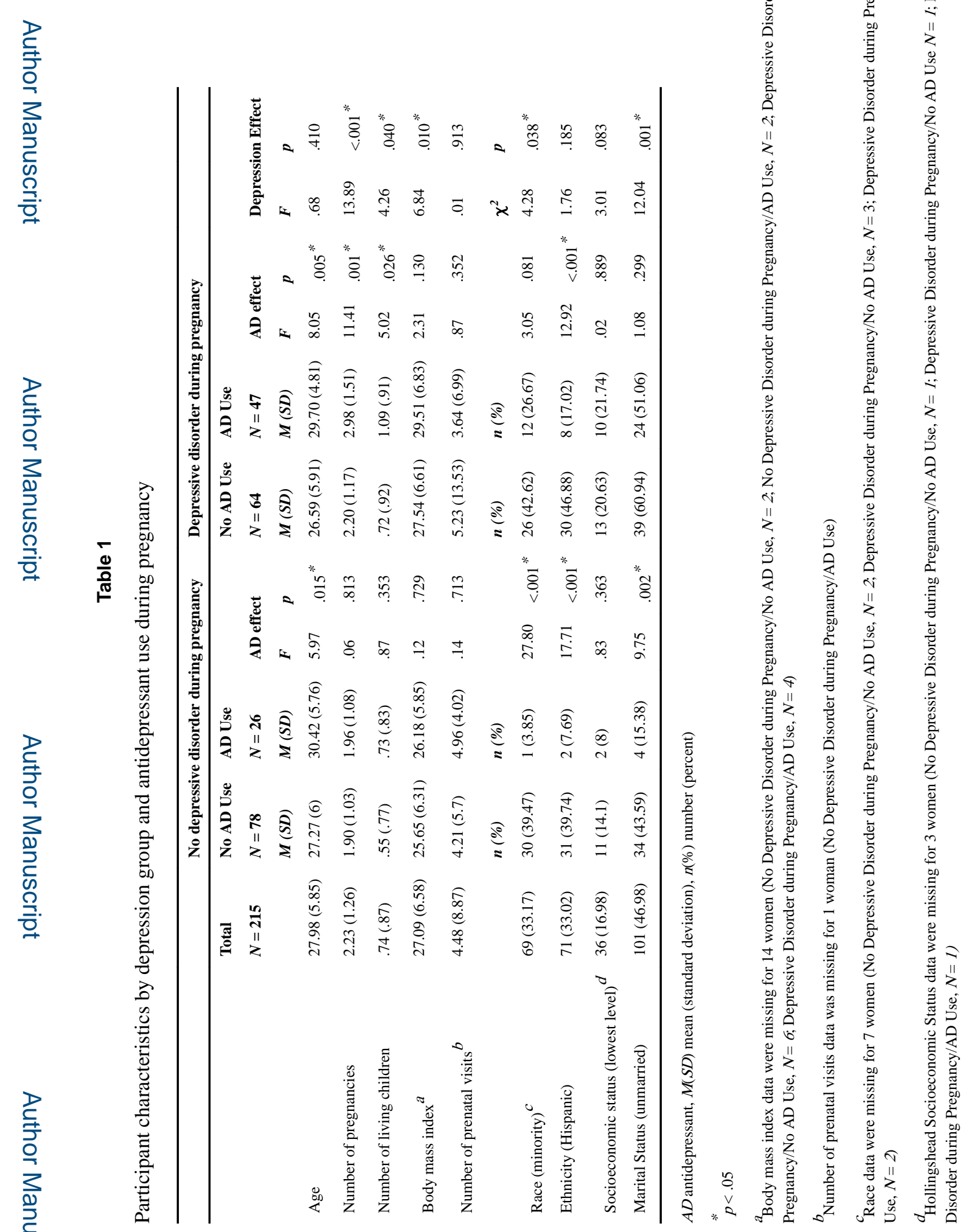




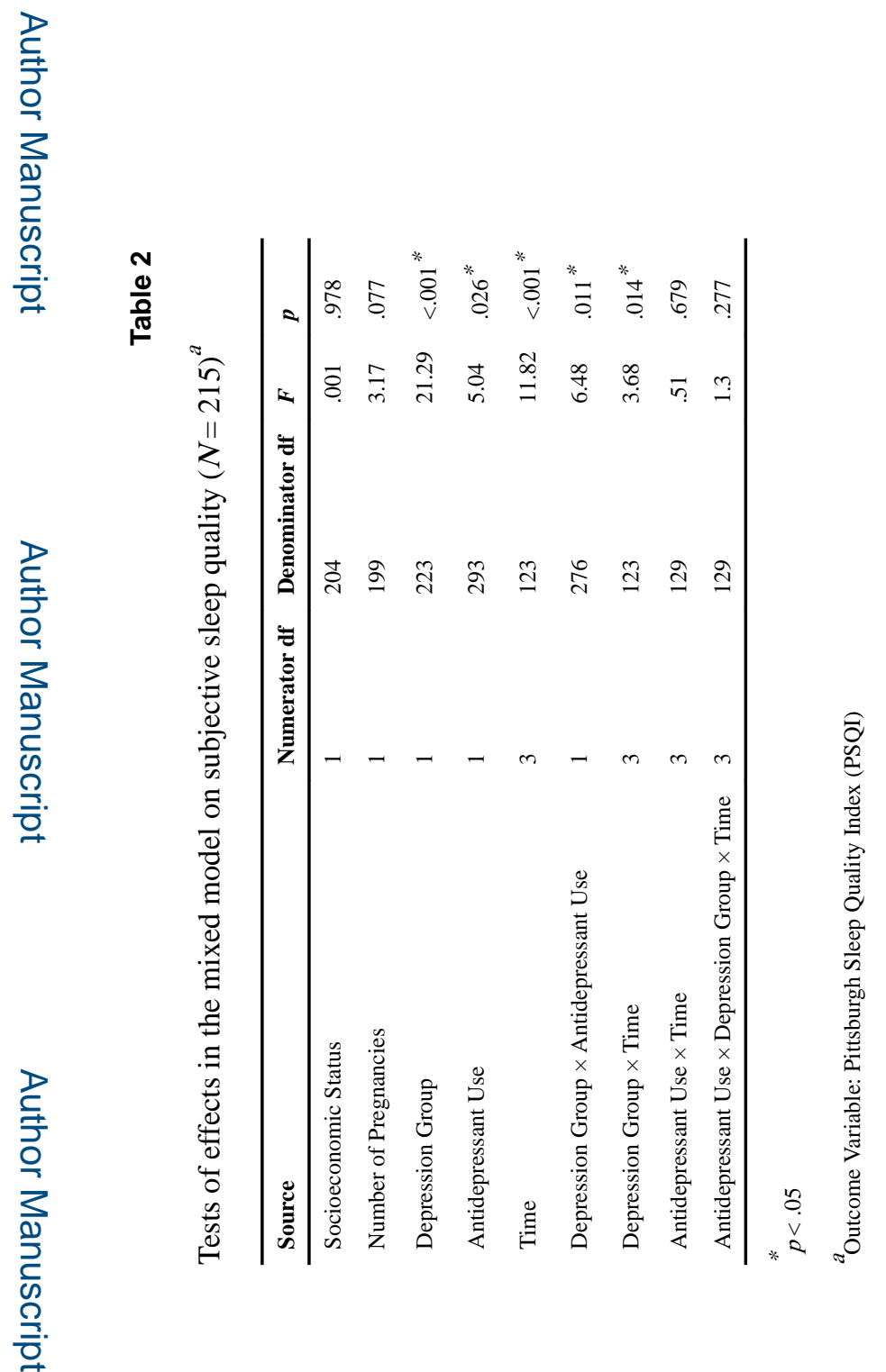




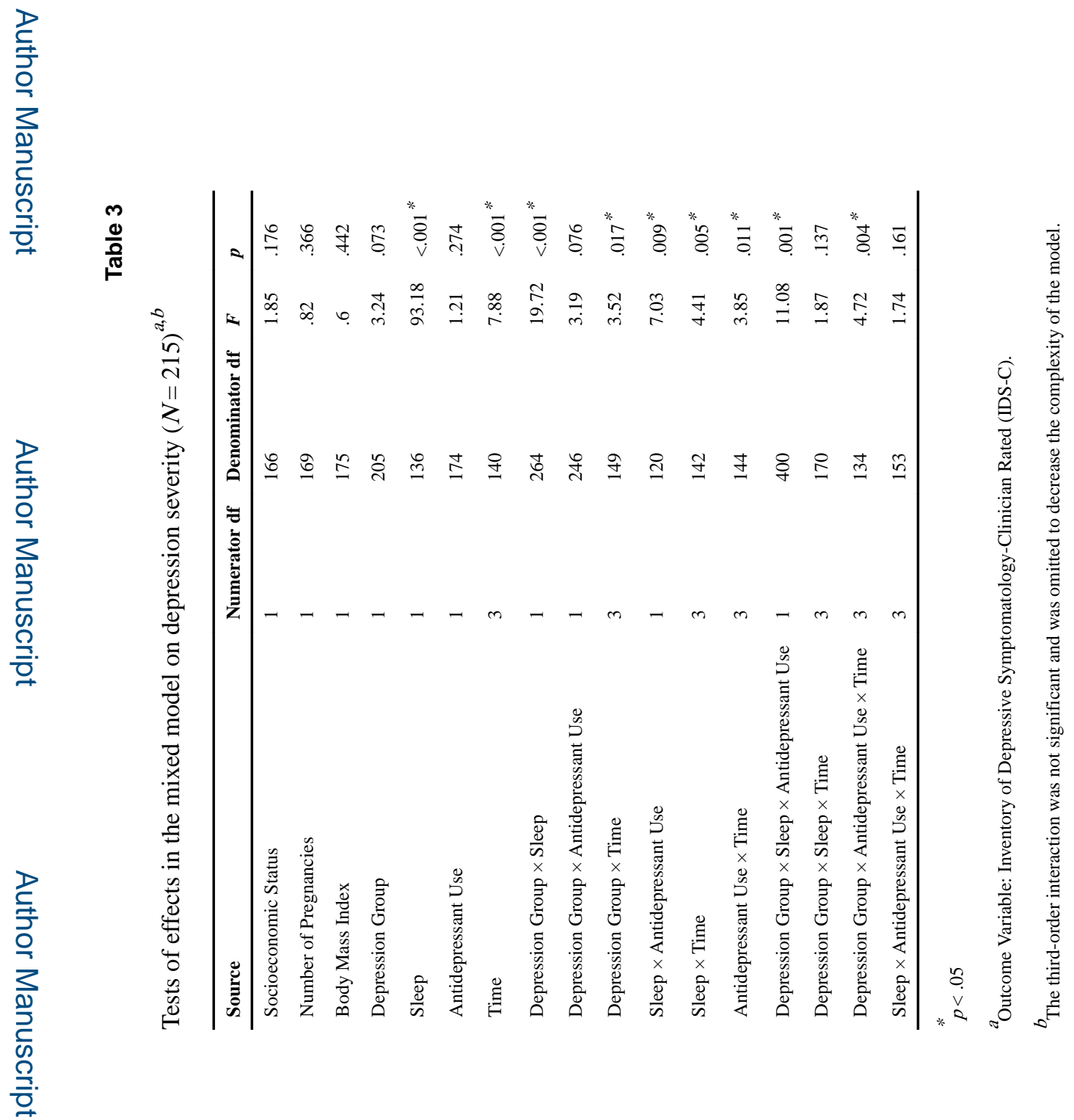

